\title{
Constraints on the cosmic ray cluster physics from a very deep observation of the Perseus cluster with MAGIC
}

\author{
Pierre Colin* \\ Max-Planck-Institut für Physics, Munich, Germany \\ E-mail: colin@mppmu.mpg.de
}

\section{Fabio Zandanel}

GRAPPA Institute, University of Amsterdam, The Netherlands

E-mail: f.zandanel@uva.nl

\section{Monica Vazquez Acosta ${ }^{a}$, Joaquim Palacio ${ }^{b}$ for The MAGIC collaboration,}

${ }^{a}$ Instituto de Astrofisica de Canaria, La Laguna (Tenerife), Spain

${ }^{b}$ Institut de Fisica d'Altes Energies, Bellaterra (Barcelona), Spain

\section{Christoph Pfrommer ${ }^{c}$ and Anders Pinzke ${ }^{d}$}

${ }^{c}$ HITS, Schloss-Wolfsbrunnenweg 33, Heidelberg, Germany

${ }^{d}$ Dark Cosmology Center, University of Copenhagen, Denmark

\begin{abstract}
Galaxy clusters are the largest and most massive gravitationally bound structures known in the Universe. Cosmic-Ray (CR) hadrons accelerated at structure formation shocks and injected by galaxies, are confined in galaxy clusters where they accumulate for cosmological times. The presence of diffuse synchrotron radio emission in several clusters proves the existence of high-energy electrons, and magnetic fields. However, a direct proof of CR proton acceleration is missing. The presence of CR protons can be probe through the diffuse $\gamma$-ray emission induced by their hadronic interaction with the Intra-Cluster Medium (ICM). The Perseus cluster, a nearby cool-core cluster, has been identified to be among the best candidates to detect such emission. We present here the results of a very deep observation of the Perseus cluster with the MAGIC telescopes, accumulating about 250 hours of data from 2009 to 2014. No evidence of large-scale very-high-energy $\gamma$-ray emission from CR-ICM interactions has been detected. The derived flux upper limits in the $\mathrm{TeV}$ regime allow us to put stringent constraints on the physics of cluster CRs, in particular on the CR-to-thermal pressure, the CR acceleration efficiency at formation shocks and the magnetic field of the central cluster region.
\end{abstract}

The 34th International Cosmic Ray Conference,

30 July- 6 August, 2015

The Hague, The Netherlands

\footnotetext{
* Speaker.
} 


\section{Introduction}

Clusters of galaxies represent the latest stage of the structure formation, produced by merging small group of galaxies and gas accretion. Their study is a powerful cosmological tools to test the evolution model of the Universe [1]. Cosmic-ray (CR) protons can be accelerated by structure formation shocks, and outflows from galaxies and active galactic nuclei (AGNs) of the cluster, and accumulate in it for cosmological times. These CR protons can interact hadronically with the protons of the intra-cluster medium (ICM), a hot thermal plasma with $k_{B} T \sim \mathrm{keV}$, and generate pions. While $\pi^{ \pm}$'s decay to secondary electrons and neutrinos, the $\pi^{0}$ 's decay directly to high-energy $\gamma$ rays. Despite many observational efforts, diffuse $\gamma$-ray emission from clusters has remained undetected.

Non-thermal emission is observed at radio frequencies in many galaxy clusters in the form of diffuse synchrotron radiation [2]. This probes the presence of relativistic CR electrons and magnetic fields in the cluster environment. But, a proof for CR-proton acceleration has yet to be found. The observed diffuse radio emission in clusters can be divided in three main categories: peripheral radio relics, central radio halos and mini-halos $[2,3]$. While radio relics are though to be related to merger shocks, the origin of radio halos and mini-halos have been historically debated between re-acceleration and hadronic models. In the re-acceleration model, a seed population of CR electrons is re-accelerated by turbulence, while in the hadronic scenario the radio-emitting electrons are secondaries produced by CR protons interacting with the ICM. Actually, the presence and role of CR protons in clusters can be probed directly only through the $\gamma$-rays and neutrinos induced by hadronic interactions. The high-energy astronomy window is then crucial to understand non-thermal phenomena in galaxy clusters.

The Perseus cluster of galaxies (Abell 426) is a relaxed, cool-core cluster located at a distance of about $78 \mathrm{Mpc}(z=0.018)$. It hosts the brightest thermal X-ray emission from the ICM [7] and a very luminous radio mini-halo $[4,5]$. The high ICM density at the centre implies a high target density for CR hadronic interactions. Therefore, Perseus is the best cluster where to search for CRinduced $\gamma$-ray emission (see $[8,9,10,11]$ for a detailed argumentation). This cluster also hosts two $\gamma$-ray bright AGNs: NGC 1275, the central dominant galaxy of the cluster, and IC 310, a peculiar object that could be an intermediate state between a BL Lac and a radio galaxy. Both were detected with Fermi-LAT [12, 13] and MAGIC [14, 15].

Since 2008, the Perseus cluster is intensively observed by the MAGIC telescopes. Here, we present the results obtained with $\sim 250$ hours of effective observation time taken in stereoscopic mode from 2009 to 2014, where constraints on the CR population in the cluster are derived.

\section{MAGIC observations and results}

MAGIC is a system of two $17 \mathrm{~m}$ diameter Imaging Atmospheric Cherenkov Telescopes located on the Canary island of La Palma. It observes the $\gamma$-ray sky from $\sim 50 \mathrm{GeV}$ to more than $50 \mathrm{TeV}$. The observation of the Perseus cluster started in 2008 with $\sim 24 \mathrm{~h}$ of observation with a single telescope, that did not allow any detection [9]. Since 2009, MAGIC operates in stereoscopic mode, providing a much better sensitivity [17]. Here, we combine all the Perseus-cluster stereo observations carried out from October 2009 to November 2014. The observations of a first period, 

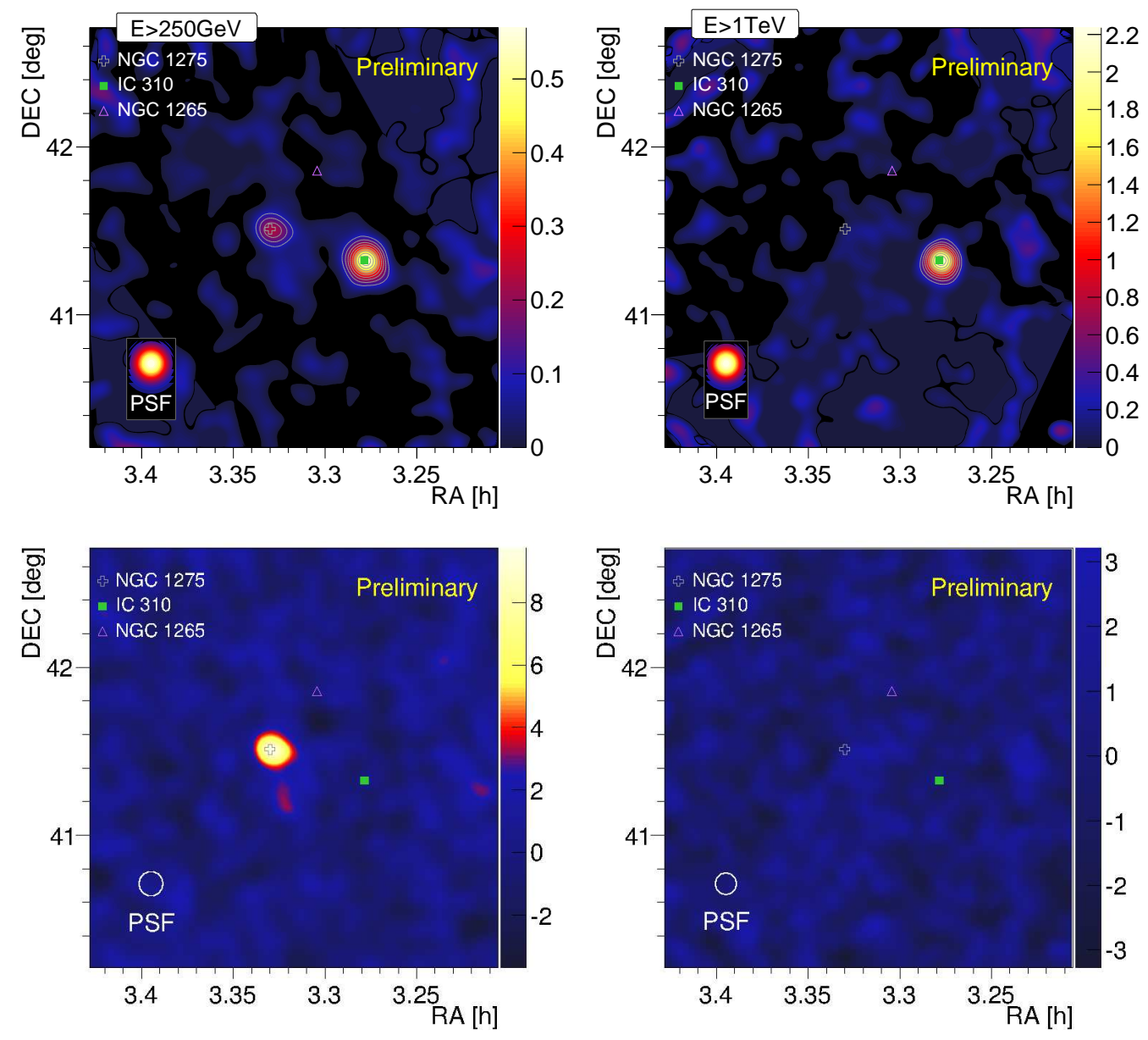

Figure 1: Perseus cluster sky maps for an energy threshold of $250 \mathrm{GeV}$ (left-hand panels) and $1 \mathrm{TeV}$ (righthand panels) obtained from $\sim 250 \mathrm{~h}$ of MAGIC observation. Top panels show the relative flux (colour code, expressed in signal-to-background ratio) and the excess significance (contour lines starting from $4 \sigma$ with steps of $2 \sigma$ ). Lower panels show the significance maps where the signal from IC 310 is included in the background model. Symbols indicate the positions of the three brightest radio galaxies of the cluster.

2009-2011, taken solely during dark time at low zenith angle (from $12^{\circ}$ to $36^{\circ}$ ), led to the discovery of IC 310 [14] and NGC 1275 [15]. The observations of the period 2012-2014 were carried out after an upgrade of the telescopes [18]. They were taken with relaxed criteria including Moonlightnight and a larger zenith-angle coverage from $12^{\circ}$ to $60^{\circ}$. After data-quality selection, the final sample in the two periods considered amounted to an effective time of $85 \mathrm{~h}$ and $168 \mathrm{~h}$, respectively.

The standard MAGIC Analysis and Reconstruction Software (MARS) is used to analyze the data. For the 2009-2011 data sample, we use the same analysis than in our previous study on cluster CR-physics [11]. For the 2012-2014 data sample, to properly handle the data taken under Moonlight we use 33\% higher image cleaning levels than the standard levels reported in [17]. This increases the energy threshold but does not affect the performance at high energy.

The $253 \mathrm{~h}$ of stereo observation, combined at the latest stage of the analysis, provide the deepest view of the Perseus cluster at very high energy. The right- and left-upper panels of Figure 1 show 
the relative-flux (i.e., signal-to-background ratio) sky maps for an energy threshold of $250 \mathrm{GeV}$ and $1 \mathrm{TeV}$, respectively. A clear signal is detected from the two previously discovered AGNs. The bright and hard source IC 310 is visible in both maps with high significance and could mask smaller signals. In order to search for weak emissions, we included the point-like emission from IC 310 in our background model. The lower panels of Figure 1 show the resulting significance sky maps. Above $250 \mathrm{GeV}$, NGC 1275 is detected with a significance $>8 \sigma$. The shape of the detected signal is in perfect agreement with a point-like object such as expected for an AGN. Above $1 \mathrm{TeV}$, however, no source other than IC 310 is detected. For both energies, there is no sign of diffuse $\gamma$-ray structures inside the cluster.

The average energy spectrum of NGC 1275 obtained with the full 2009-2014 data set is shown in Figure 2, together with the previously reported spectra from the first two years of observation [16]. The spectrum of this analysis starts at higher energy because the data include Moonlight and large-zenith-angle observations. The statistical precision is largely improved, extending the spectrum coverage with a data point around $880 \mathrm{GeV}$. The spectrum between $90 \mathrm{GeV}$ and $1200 \mathrm{GeV}$ can be described by a simple power law

$$
\frac{\mathrm{d} F}{\mathrm{~d} E}=f_{0}\left(\frac{E}{200 \mathrm{GeV}}\right)^{-\Gamma}
$$

with a photon index $\Gamma=3.6 \pm 0.2_{\text {stat }} \pm 0.2_{\text {syst }}$ and a normalization constant at $200 \mathrm{GeV}$ of $f_{0}=$ $\left(2.1 \pm 0.2_{\text {stat }} \pm 0.3_{\text {syst }}\right) \times 10^{-11} \mathrm{~cm}^{-2} \mathrm{~s}^{-1} \mathrm{TeV}^{-1}$.

\section{Search for cosmic-ray induced emission}

The $\gamma$-ray emission from the central galaxy NGC 1275 is consistent with a point-like source and no diffuse component is observed. The measured flux is much larger than what is expected from the CR-induced emission, and it could be outshining the latter. However, the NGC 1275 AGN spectrum is very steep and no signal is detected above about $1 \mathrm{TeV}$, while models expect the CRinduced spectrum to be a flat power-law with no cutoff in the MAGIC energy range [8]. Therefore, we use the energies above $1 \mathrm{TeV}$ to search for the possible diffuse CR-induced component.

As the spatial shape of the CR-induced emission is not known, three different models are used as template: i) the isobaric model assuming a constant CR-to-thermal pressure $X_{\mathrm{CR}}=P_{\mathrm{CR}} / P_{\mathrm{th}}$ [20], ii) the semi-analytical model for CRs derived from hydrodynamical simulations of clusters [8], and iii) the extended hadronic model of [21], where the possibility of CR propagation out of the cluster core is considered, resulting in a significantly flatter CR profile. The $\gamma$-ray surface brightness of these three models is shown in Figure 3. The fraction of signal expected within a circular region of a given radius $\theta$ from the cluster centre and the MAGIC Point Spread Function (PSF) above $630 \mathrm{GeV}$ are also shown. Since the predicted CR-induced signal extension is significantly larger than the PSF, optimal selection cuts $\theta_{\text {cut }}$, different than for a point-like source, are used to detect the emission for each of the models, which are shown in Figure 3.

To derive the strongest possible constraints, we compute upper limits on the integral flux above a given energy that accumulates results of several energy bins. The obtained limits depend on the assumed spectral shape. We adopt the universal CR-proton spectrum found with hydrodynamical simulations [8], that suggests a power-law momentum spectrum $p^{-\alpha}$ with $\alpha \approx 2.2$ at the energies 


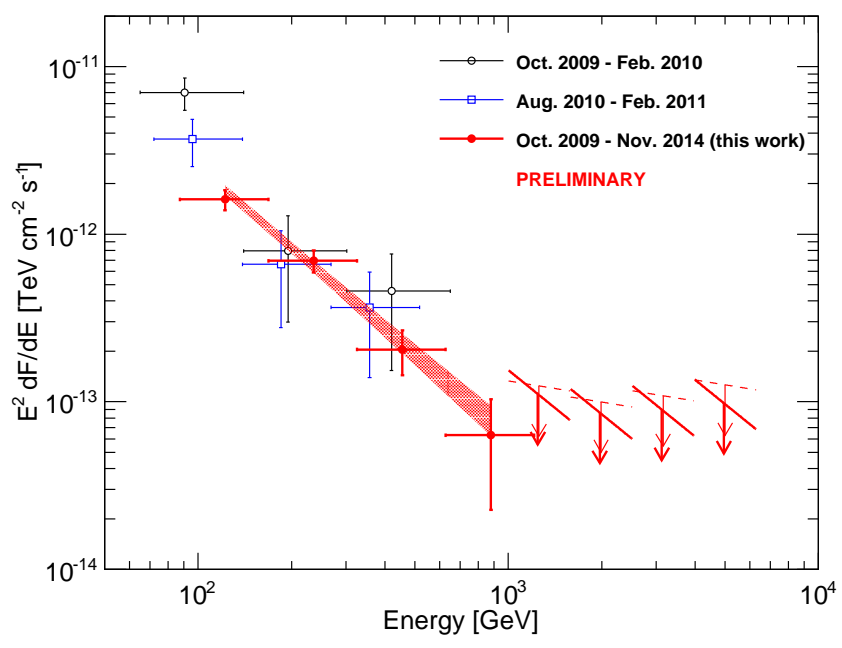

Figure 2: Spectral energy distribution of NGC 1275 averaged over different periods. The red symbols show our new result obtained with $\sim 250 \mathrm{~h}$ of data. The red arrows are the differential flux upper limits for a power-law spectrum with a photon index $\Gamma=3.5$ (thick solid lines) and $\Gamma=2.3$ (thin and dashed lines).
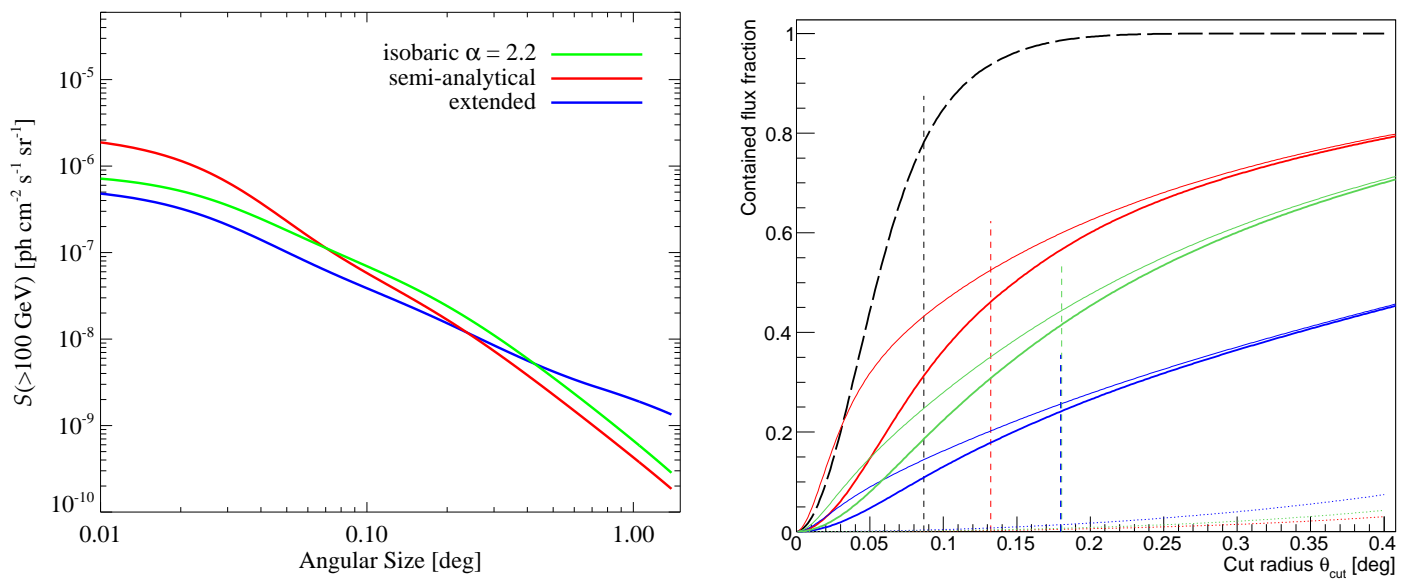

Figure 3: Left: Surface brightness profiles of the three tested spatial templates for the CR-induced emission in Perseus. Right: Cumulative fraction of signal within a given radius for different models: point-like in black (long-dashed line), isobaric in green, semi-analytical in red, and extended in blue. The distribution of the point-like model corresponds the the MAGIC PSF above $630 \mathrm{GeV}$. The thin and thick coloured solid lines represent the real and MAGIC-reconstructed, i.e., smeared by the PSF, signal fractions, respectively. The dotted lines show the fraction of these signals contained in the background region. The vertical dashed lines correspond to the used optimal $\theta_{\text {cut }}$ values.

of interest here. At very high energy, the $\gamma$-ray spectrum induced by pion decays should have approximately the same spectral index. We convert this intrinsic spectrum into the spectrum observed on Earth, taking into account the $\gamma$-ray absorption by the Extragalactic Background Light (EBL) [22] from the Perseus cluster at $z=0.018$. Between $300 \mathrm{GeV}$ and $10 \mathrm{TeV}$, the effect of the absorption can be approximated by an increase (softening) of the power-law index of about 0.13 and a reduc- 
Table 1: Integral flux upper limits within a radius of $0.15^{\circ}$ and $1.4^{\circ}$ from the Perseus cluster centre, between $E_{t h}$ and $10 \mathrm{TeV}$ for a power-law spectrum with an index $\Gamma=2.33$ [in unit of $10^{-14} \mathrm{~cm}^{-2} \mathrm{~s}^{-1}$ ].

\begin{tabular}{lcccccc}
\hline \hline$E_{t h}$ & model & ON & OFF & $\sigma_{L i M a}$ & $\mathrm{~F}_{U L}^{0.15^{\circ}}$ & $\mathrm{F}_{U L}^{1.4^{\circ}}$ \\
\hline \multirow{5}{*}{$630 \mathrm{GeV}$} & point-like & 332 & 304.1 & 1.4 & 12.2 & 12.2 \\
& isobaric & 1327 & 1256 & 1.4 & 24.8 & 65.5 \\
& semi-analytic & 749 & 681 & 1.8 & 26.8 & 49.0 \\
& extended & 1327 & 1256 & 1.4 & 25.6 & 124. \\
\hline \multirow{3}{*}{$1.0 \mathrm{TeV}$} & point-like & 159 & 157.5 & 0.1 & 3.84 & 3.84 \\
& isobaric & 675 & 652 & 0.6 & 10.7 & 28.3 \\
& semi-analytic & 369 & 352 & 0.6 & 9.77 & 17.9 \\
& extended & 675 & 652 & 0.6 & 11.1 & 54.0 \\
\hline \multirow{3}{*}{$1.6 \mathrm{TeV}$} & point-like & 77 & 75.9 & 0.1 & 2.34 & 2.34 \\
& isobaric & 321 & 317 & 0.2 & 5.09 & 13.5 \\
& semi-analytic & 169 & 167 & 0.1 & 4.61 & 8.43 \\
& extended & 321 & 317 & 0.2 & 5.26 & 25.6 \\
\hline
\end{tabular}

Notes. ON and OFF are the number of events in the signal (ON) and background (OFF) regions, respectively. $\sigma_{L i M a}$ is the significance of the excess expressed in standard deviations.

tion of the differential flux at $1 \mathrm{TeV}$ of $17 \%$. Above $10 \mathrm{TeV}$ the absorption increases dramatically. A source with an intrinsic spectral index $\alpha=2.2$ would appear above $300 \mathrm{GeV}$ as a power-law with an index $\Gamma=2.33$ and a cutoff above $10 \mathrm{TeV}$. About $20 \%$ of the flux above $1 \mathrm{TeV}$ and $60 \%$ above $10 \mathrm{TeV}$ is absorbed during the travel to Earth. This effect was neglected in all previous papers, which, therefore, overestimate the constrains induced by the flux upper limit.

Table 1 presents the 95\%-confidence-level upper limits (method from [19] with a total systematic uncertainty of $30 \%$ ) of the integral flux between several energy thresholds $E_{t h}$ and $10 \mathrm{TeV}$ for different models assuming a power-law spectrum with $\Gamma=2.33$. The upper limits are converted to the corresponding flux contained within the reference radius $0.15^{\circ}$ and the cluster virial radius ${ }^{1} R_{200} \simeq 1.4^{\circ}$ [23]. The conversion factors depend on the surface brightness distributions, considering also the signal contamination in the OFF region.

\section{Interpretation and discussion}

One of the main uncertainties in modeling CR physics in clusters of galaxies is the CR spatial distribution, which is assessed by considering different spatial templates (isobaric, semi-analytical and extended). Another major uncertainty is the CR-acceleration efficiency, i.e., the fraction of the energy dissipated at structure formation shocks which causes particle acceleration. Our semianalytical model is based on predictions by [8] derived from hydrodynamical simulations of clusters, which assume a maximum CR-proton acceleration efficiency $\zeta_{\mathrm{p}, \max }=50 \%$. Here, we tie this

\footnotetext{
${ }^{1}$ The cluster virial radius is here defined with respect to a density that is 200 times the critical density of the Universe.
} 
to the parameter $A_{\gamma}$, just a multiplier for the whole formalism, that is $A_{\gamma}=1$ for $\zeta_{\mathrm{p} \text {,max }}=50 \%$. Smaller values of $A_{\gamma}$ correspond to smaller values of $\zeta_{\mathrm{p} \text {,max }}$.

The left-hand panel of Figure 4, shows the integral $\gamma$-ray fluxes predicted by [8] within $0.15^{\circ}$ from the centre, $A_{\gamma}=1$, and the highest fluxes allowed by our observational upper limits. Compared to [11], we accumulate three times more data and derive upper limits that are significantly lower. $A_{\gamma}$ is constrained to be $\leq 0.56$ when neglecting the EBL absorption and $\leq 0.75$ with the EBL[22]. This corresponds to a maximum CR-proton acceleration efficiency of $28 \%$ and $37 \%$, respectively. The latter should be considered the reference value while the one without EBL absorption is given to allow comparison to previous results.
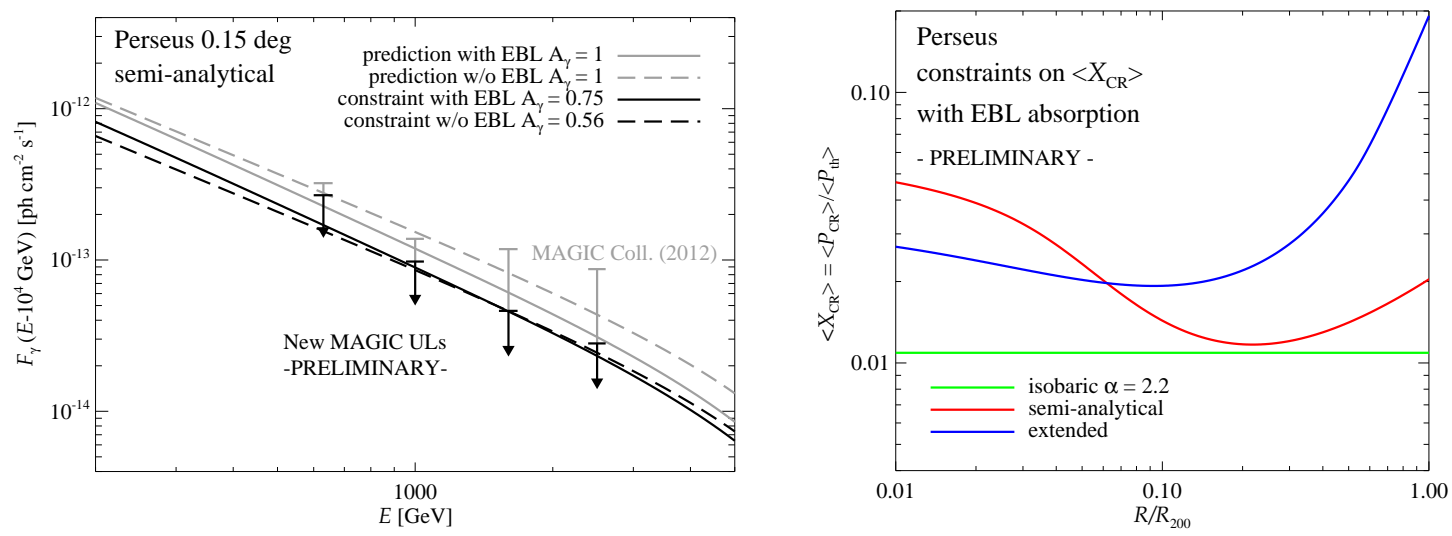

Figure 4: Left. Integral flux upper limits and spectra within $0.15^{\circ}$ for the semi-analytical model. We show the spectrum predicted by [8] and the ones adapted to match our upper limits. Shown in light gray are our previous flux upper limits from [11]. Right. Volume-averaged $X_{\mathrm{CR}}$ within a given radius $R$, as constrained by the upper limits presented in this work for the semi-analytical, extended, and isobaric models with $\alpha=2.2$.

The constraints on the volume-averaged CR-to-thermal pressure profiles for the three CRdistribution models are shown in Figure 4-left. $\left\langle X_{\mathrm{CR}}>\right.$ results to be below $1-2 \%$ within $0.15^{\circ}$ $\left(\approx 0.11 \times R_{200}\right)$. When considering the full galaxy cluster volume up to $R_{200},<X_{\mathrm{CR}}>$ is below $2 \%$ for the isobaric and semi-analytical models, but significantly less constrained, below $19 \%$ for the extended model. This latter weak constraint was expected because the CR distribution is significantly flatter in this case.

The Perseus cluster hosts a bright radio mini-halo. Assuming the hadronic model of radio halos, i.e., synchrotron emission generated by secondary electrons produced in hadronic interactions between the CR and ICM, the pion-decay $\gamma$-ray emission is directly linked to radio signal. The intensity of the synchrotron emission depends on the amount of electrons, proportional to the hadronically-induced $\gamma$ rays, and the local magnetic field. This emission can be reduced due to the electron energy lost through other channels, mainly inverse Compton. In the regime of very strong magnetic fields, the electrons lose all their energy through synchrotron and we can derive the expected $\gamma$-ray emission. For assumptions of CR spectral index $\alpha \leq 2.1$, the expected $\gamma$-ray emission is above our upper limits. For softer CR spectrum, our $\gamma$-ray upper limits can be turned into lower limits on the magnetic field. This constraint, depending on the photon field considered for the inverse Compton scattering, is still under study. 


\section{Acknowledgment}

We would like to thank the Instituto de Astrofísica de Canarias for the excellent working conditions at the Observatorio del Roque de los Muchachos in La Palma. The financial support of the German BMBF and MPG, the Italian INFN and INAF, the Swiss National Fund SNF, the ERDF under the Spanish MINECO (FPA2012-39502), and the Japanese JSPS and MEXT is gratefully acknowledged. This work was also supported by the Centro de Excelencia Severo Ochoa SEV-2012-0234, CPAN CSD2007-00042, and MultiDark CSD2009-00064 projects of the Spanish Consolider-Ingenio 2010 programme, by grant 268740 of the Academy of Finland, by the Croatian Science Foundation (HrZZ) Project 09/176 and the University of Rijeka Project 13.12.1.3.02, by the DFG Collaborative Research Centers SFB823/C4 and SFB876/C3, and by the Polish MNiSzW grant 745/N-HESS-MAGIC/2010/0.

\section{References}

[1] G. M. Voit, Reviews of Modern Physics 77, 207 (2005)

[2] L. Feretti, G. Giovannini, F. Govoni, and M. Murgia, A\&Ar 20, 54 (2012)

[3] G. Brunetti and T. W. Jones, International Journal of Modern Physics D 23, 30007 (2014)

[4] A. Pedlar et al., MNRAS 246, 477 (1990)

[5] L. G. Sijbring, PhD thesis, Groningen University (1993)

[6] T. Enßlin, C. Pfrommer, F. Miniati, and K. Subramanian, A\&A 527, 99 (2011)

[7] E. Churazov, W. Forman, C. Jones, and H. Böhringer, ApJ 590, 225 (2003)

[8] A. Pinzke and C. Pfrommer, MNRAS 409, 449 (2010)

[9] MAGIC Collaboration, Aleksić et al., ApJ 710, 634 (2010)

[10] A. Pinzke, C. Pfrommer, and L. Bergström, Phys. Rev. D, 84, 123509 (2011)

[11] MAGIC Collaboration, Aleksić et al., A\&A 541, 99 (2012)

[12] Fermi-LAT Collaboration, A. A. Abdo et al., ApJ 699, 31 (2009)

[13] A. Neronov, D. Semikoz, and I. Vovk, $A \& A$ 519, L6 (2010)

[14] MAGIC Collaboration, Aleksić et al., ApJ 723, L207 (2010)

[15] MAGIC Collaboration, Aleksić et al., A\&A 539, L2 (2012)

[16] MAGIC Collaboration, Aleksić et al., A\&A 564, 5 (2014)

[17] MAGIC Collaboration, Aleksić et al., Astroparticle Physics in press [arXiv1409.5594], (2015)

[18] MAGIC Collaboration, Aleksić et al., Astroparticle Physics in press [arXiv1409.6073], (2015)

[19] W. A. Rolke, A. M. López, and J. Conrad, NIM A 551, 493 (2005)

[20] C. Pfrommer and T. A. Enßlin, $A \& A$ 413, 17 (2004)

[21] F. Zandanel, C. Pfrommer, and F. Prada, MNRAS 438, 124 (2014)

[22] A. Domínguez et al. Astroparticle Physics 410, 2556 (2011)

[23] T. H. Reiprich and H. Böhringer, ApJ 567, 716 (2002) 